EPJ Web of Conferences 66, 10008 (2014)

DOI: 10.1051/epjconf/ 20146610008

(C) Owned by the authors, published by EDP Sciences, 2014

\title{
Fukushima Nuclear Power Plant Accident and Nuclear Physicists
}

\author{
Takaharu Otsuka ${ }^{1,2, a}$ \\ ${ }^{1}$ Department of Physics and Center for Nuclear Study, University of Tokyo, Hongo, Bunkyo-ku, Tokyo, 113- \\ 0033, Japan \\ ${ }^{2}$ National Superconducting Cyclotron Laboratory and Department of Physics and Astronomy, Michigan State \\ University, East Lansing, MI 48824, U.S.A.
}

\begin{abstract}
I give an overview on the Fukushima Nuclear Power Plant Accident and a report on voluntary activities of Japanese nuclear physicists in this terrible event, including their major outcome.
\end{abstract}

\section{Introduction}

I report, in this talk, some of the activities carried out by Japanese nuclear physicists in response to Fukushima Dai-ichi Nuclear Power Plant accident caused by the big earthquake on March 11, 2011. Although there were many activities conducted by volunteers from the nuclear physics community in Japan, I would like to focus on the monitoring of radioactivity from human beings and the survey of soil contamination by fallen radioactive materials. Let me start with a brief summary of what happened.

\section{The earthquake and Tsunami}

The earthquake has started at 14:46:18 on the 11th of March, 2011, and turned out to be the biggest one in the documented history of Japan. It was not a single earthquake actually, but a series of earthquakes. The magnitude was 9.0, which is weaker only by 0.1 than the famous Sumatra earthquake in 2004 . The initial earthquake started about $130 \mathrm{~km}$ in the east of Oga peninsula in Miyagi prefecture (to which Sendai is the local capital), and the subsequent earthquakes took place in a wide area of 500 $\mathrm{km}$ (north-south) times $200 \mathrm{~km}$ (east-west). Two plates under the Pacific ocean slid against each other by 30-60 meters at maximum. This enormous movement at the bottom of the ocean produced huge Tsunami on its surface.

Due to the earthquake and Tsunami, 18,564 people are dead or remain missing (as of the time of the INPC 2013). Economic loss was estimated to be $16-25 \times 10^{12}$ Yen, which is equivalent to $1.2-2 \times$ $10^{10}$ Euros.

There was no major mechanical damage to the Fukushima Dai-ichi Nuclear Power Plant of Tokyo Electric Power Corporation (TEPCO) according to the report of the government investigation committee of the accident, as well as to other sources. However, there was a fatal miscalculation by TEPCO.

\footnotetext{
a e-mail: otsuka@phys.s.u-tokyo.ac.jp
} 
This was the complete loss of the external electric power supply to the power plant. By the earthquake, one of the towers suspending electric high-voltage cables has fallen due to the land slide, because it was on a hill. Such complete loss of the electric power supply from the outside (the power plant) may be one of what the TEPCO did not anticipate. The nuclear power plants reacted to the earthquake properly, and chain reactions have been stopped, as the control rods have come down as a step of the emergency procedure. This means also that the electricity produced by the nuclear reactors was gone completely. The emergency power generators with diesel engines started to work as planned, and the electric power supply was maintained at this stage. The situation proceeded up to this point quite well according to the emergency plan.

Here, let me overview very briefly what Fukushima prefecture is. It is one of the largest prefectures of Japan. Its local capital is called also Fukushima, located about $200 \mathrm{~km}$ in the north of Tokyo. Although this distance is not so much, Fukushima prefecture is rather rural area with lower density of population. On the other hand, it has a long coast line. Because of these reasons combined, there are many power plants in Fukushima's coast: 10 nuclear, 4 oil burning and 2 coal burning, owned by TEPCO.

\section{Fukushima Dai-ichi Nuclear Power Plant}

The Fukushima Dai-ichi Nuclear Power Plant has six reactors, No. 1-6. Tsunami came to the plant around 16:00, which is about an hour after the first impact of the earthquake. The height of Tsunami was 13.1 meters at maximum, which turned out to be much higher than the bank prepared against waves up to 5.7 meters high. This was another miscalculation by TEPCO. There were some warnings for higher Tsunami before the earthquake. No strong convincing message, however, had been issued from the community of geoscience, or TEPCO did not take the warnings seriously. This is quite unfortunate, partly because one geoscientist employed by TEPCO made such a warning but the top management of TEPCO was not aware of it. Such management problems can be one of the causes of the accident.

Because of the Tsunami, the areas of reactors No. 1-4 were covered by sea water. Tsunami did not damage the reactors directly, but the sea water flew down to the emergency generators placed on the basement. All emergency generators of reactors No. 1-4 were stopped. It was not a wise idea to install emergency generators on the basement, if one considers the possibility of Tsunami. The reactors No. 1-4 have been operational since 1971, 74, 76 and 78, respectively. They are not new. In fact, the reactor No. 1 is the first reactor owned and used by commercial electricity company in Japan. So, the design was made primarily according to US standards. In the US, the hurricane is the most dangerous natural disaster, and the basement is the safest place against strong winds by hurricanes. Typhoon in Japan causes strong wind, but it is not as terrible as Hurricane's. On the other hand, Tsunami is quite dangerous.

No. 5 and 6 reactors of Fukushima Dai-ichi Plant are located slightly away and higher, and have managed to survive. There are nuclear reactors also in Miyagi prefecture, and have survived because of higher elevation too.

The type of reactors No. 1-4 is Boiling Water Reactors (BWR) with power 460 MWe for No. 1 and 784 MWe for No. 2-4. They are not among the biggest models, but are somewhat old.

The loss of the external electric power and the loss of the emergency generators mean that there is no working pumps for the cooling water for reactors or storage pools of fuel rods, in long terms. The batteries cannot supply sufficient electricity for more than several days. Note that even after the chain reaction is stopped, fission products in the fuel rods produce heat by $\beta$ decays, and this heat must be removed. Otherwise, the fuel rod becomes too hot, and melts. If the fuel rod melts, radioactive 
materials contained in the rod come out to the water and sink down to the bottom of the reactor container. The so-called melt down could thus happen, and is believed to have happened. Note that this is different from another type of melt-down where uncontrolled chain reactions occur in nuclear fuel. There is no evidence of this type so far.

If the fuel rod becomes hot, another problem occurs. The fuel rod is metal tube made from Zirconium. At temperature high enough, Zirconium reacts with water to produce hydrogen gas. The hydrogen gas becomes vaporized to the top of the reactor container and leaks further to other places. It can explode in certain condition, and did.

The events have started already on the first day. Around 20:30, reactor No. 2's cooling system has stopped as the battery was gone. Later on the same day, the fuel melting was feared for this reactor by the Nuclear and Industrial Safety Agency (NISA). On the 12th of March, the first hydrogen-gas explosion occurred in reactor No.1, followed by two more explosions. At night of the 12th of March, sea water was started to be injected into reactor No. 1 for the cooling purpose, but it was too late. There were many technical difficulties for the emergency measures, and a huge amount of efforts have been made by engineers on site. Nevertheless, TEPCO's insufficient preparation against severe accidents is clear, for instance, regarding preventive device against hydrogen-gas explosion, filter of radioactive materials contained in outgoing air, etc. Although I shall stop the description of the accident here, I point out that there was no explosion of the reactor itself, in contrast to Chernobyl, and the radioactive materials (fission products) leaked from damaged fuel rods into the water inside the reactor, and tiny particles carrying them went outside with the air flow. Certainly these things should have been prevented by proper devices to a great extent. Detailed studies of the accident and the processes of radioactive contamination of the environments are under way.

\section{Radioactive materials from the Fukushima nuclear accident}

Among radioactive materials leaked from the reactors, the major concern has been on ${ }^{131} \mathrm{I}$, and ${ }^{134,137}$ Cs. They are created as a result of the fission of Uranium and subsequent $\beta$ decays. As mentioned above, they were attached to tiny particles, which were flown by the wind of the air. There were two major directions. One is the northwest direction from the Power Plant, and the other is the southsouthwest. We shall show those directions later on maps. These particles came down to the earth surface when trapped by rain drops, although the precise process is to be finalized. Otherwise, they just kept flying in the sky. Thus, the amount of the contamination at a particular location was determined mainly by the combination of the wind (direction and speed) and the rain. The contamination was not uniform at all and shows very complicated patterns.

The computer program SPEEDI was made well before the accident so that such air flow can be estimated and appropriate evacuation can be conducted. However, the government did not publicize the output of SPEEDI, because the input for the simulation did not have absolute magnitude due to lack of input data. This has become a serious problem later with much criticism. Japanese scientists are working now as to how to handle relevant scientific simulations at the event of severe disasters.

I mention the scale of the accident now. According to two separate reports from NISA and Nuclear Safety Commission (NSC), the total amount of ${ }^{131} \mathrm{I}$ and ${ }^{137} \mathrm{Cs}$ emitted into the air is $1.3-1.6 \times 10^{17}$ $\mathrm{Bq}$ and 1.1-1.5 $\times 10^{16} \mathrm{~Bq}$, respectively [1]. The corresponding quantities are $1.8 \times 10^{18} \mathrm{~Bq}$ and $8.5 \times$ $10^{16} \mathrm{~Bq}$, respectively, in the case of Chernobyl [1]. This implies that Fukushima accident is about 1/10 in scale compared to Chernobyl. Such observation has been confirmed by United Nations Scientific Committee on the Effects of Atomic Radiation (UNSCEAR). 


\section{Voluntary actions by nuclear physicist}

Nuclear physicists in Japan responded promptly to the Fukushima accident. While the eastern part of Japan suffered from aftershocks and the lack of electricity for a while, the western part did not suffer directly. Some nuclear physicists gathered on the 16th of March at the Research Center for Nuclear Physics (RCNP), Osaka University, and discussed what to be done. They started to send nuclear physicists to Fukushima prefecture on the 21st of March. They kept sending nuclear physicists. Those physicists participated in the monitoring of human being, inhabitants or stuff engaged in recovery work. This operation was carried out under the control of the Fukushima-prefecture local government. Besides nuclear physicists, many other people participated, like stuff from local health offices nationwide, workers in electric companies or radioactivity-related industries, etc. According to the press release by Fukushima prefecture, about 27,000 people were monitored by the 30th of March, and about $15 \%$ was carried out by nuclear physicists. According to media reports, there has been no severe contamination of human being fortunately, but the monitoring should be and is continued particularly for young generations. I note with my sincere respect that RCNP has played a very significant role as the hub for sending nuclear physicists to Fukushima area, and that Drs. Tanihata, Fujiwara, Tamii, Hosaka and others made enormous efforts.

\section{Soil contamination maps of Fukushima area}

In addition to the monitoring of human being, another important issue was the measurement of the radioactive contamination of the environment. Nuclear physicists made a significant contribution to this as well, collaborating with geoscientists who are also nuclear chemists. We founded the organization, "Nuclear Physics-Geoscience Joint Forum on Environmental Radiation" in April, 2013. The primary purpose was to measure $\gamma$ rays originated in ${ }^{131} \mathrm{I}$ and ${ }^{134,137} \mathrm{Cs}$ in the soil of Fukushima prefecture by using Germanium detectors.

There were three major requirements:

(i) As the contamination is spread widely over the eastern part of Fukushima prefecture (and its surroundings), we must take soil samples from many locations. The survey had to be of large scale.

(ii) As the half life of ${ }^{131} \mathrm{I}$ is only 8.03 days, ${ }^{131} \mathrm{I}$ was disappearing very fast. The survey had to be quick.

(iii) As the data to be obtained were devoted to the safety and future of many people in the area, the data must be precise and reliable, in accordance with international standards on the radiation measurement.

These three points, namely, to be extensive, quick and precise, do conflict one another usually. We, however, have managed to accomplish these objectives, thanks to everyone involved.

For the point (i), we have chosen about 2200 locations. A location means one place in a $2-\mathrm{km} \times$ $2-\mathrm{km}$ mesh for the area within $80 \mathrm{~km}$ from the Fukushima plant, and one place in a $10-\mathrm{km} \times 10-\mathrm{km}$ mesh for the area outside the $80 \mathrm{~km}$ circle (but mostly still in Fukushima prefecture).

Because of point (ii), we set a two-week period by the middle of June for the sample taking operation, considering also the rainy season starting around that time every year. This means that some 700 soil samples should be taken everyday. As one team can take samples from at most 5 locations (25 samples, as explained below) per day, about 30 teams were needed. As each team consists of three scientists, many volunteers with certain knowledge were needed. A call for volunteers was made, and 409 people, including students, from 93 institutions and 3 companies responded. They have worked very hard for two weeks, and the objectives were accomplished on time. The names of these volunteers are indicated in the final report [1]. For the area inside $20 \mathrm{~km}$ from the reactors, 31 specialists from electric companies took the samples because of higher radiation. 


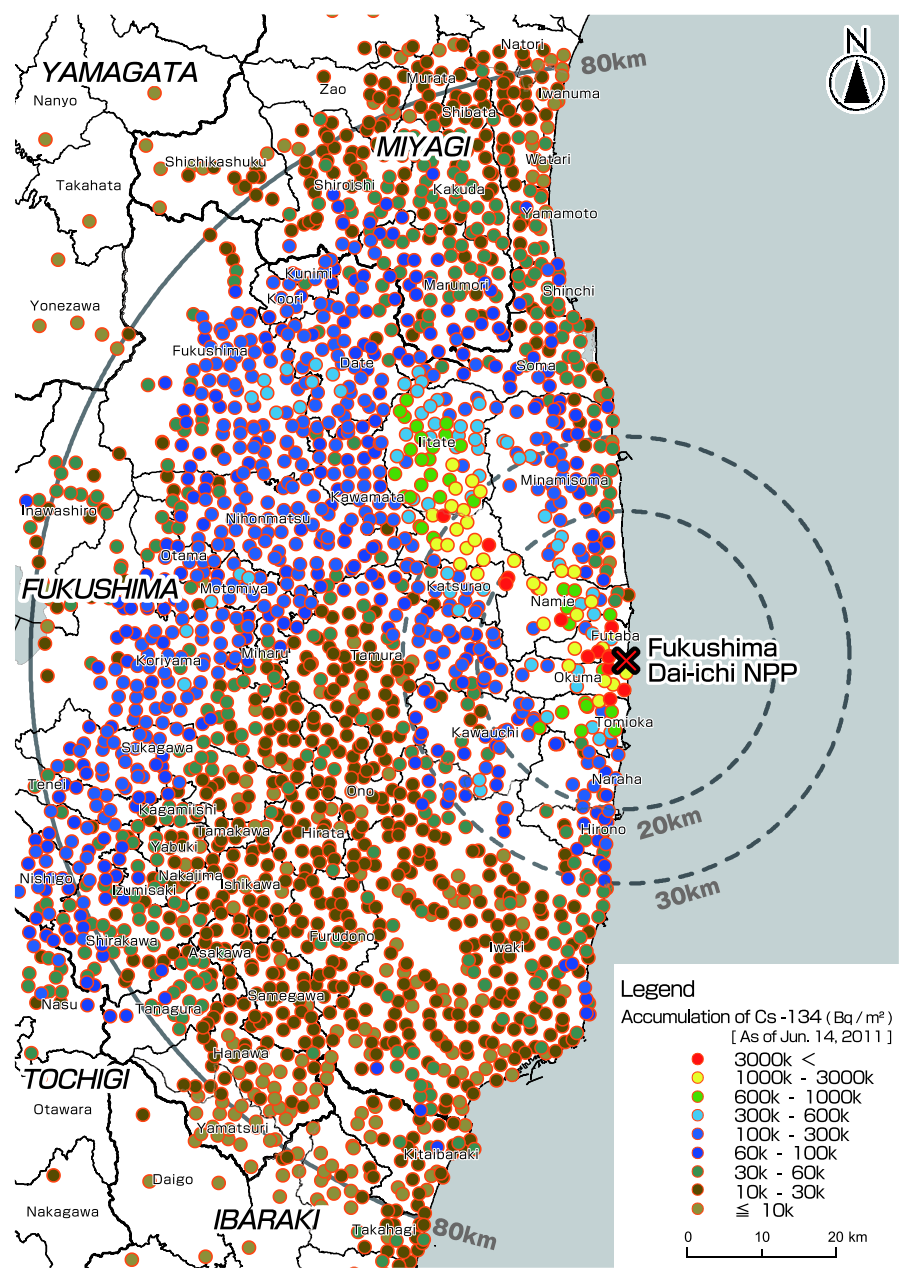

Figure 1. Contamination of soil due to ${ }^{134} \mathrm{Cs}$ per area. The unit is $\mathrm{Bq} / \mathrm{m}^{2}$.

For the point (iii), the protocol had to be established. For this purpose, some trial surveys were carried out early May, as collectively called pilot survey. The protocol has been established through such pilot surveys : five soil samples are taken from one location, the container type is fixed to so-called U-8 (100 ml plastic container, a well-established Japanese standard), and the soil in each container is mixed in a plastic bag so as to enhance the uniformity. The international approval on the protocol has been acquired mainly through the technical contract between Prof. Onda (geoscientist at the University of Tsukuba) with the Joint FAO/IAEA Programme Nuclear Techniques in Food and Agriculture.

The measurement of $\gamma$-rays needed many Germanium detectors operated by professional groups, and in fact has been carried out by the groups from 21 institutions with 343 scientists who responded to the call for the measurement. Their names are listed in the final report as well [1].

The nuclear physics community processed more than half of 11,000 soil samples. The rest was processed by Japan Chemical Analysis Center (JCAC), a corporation with the capability of radioactiv- 


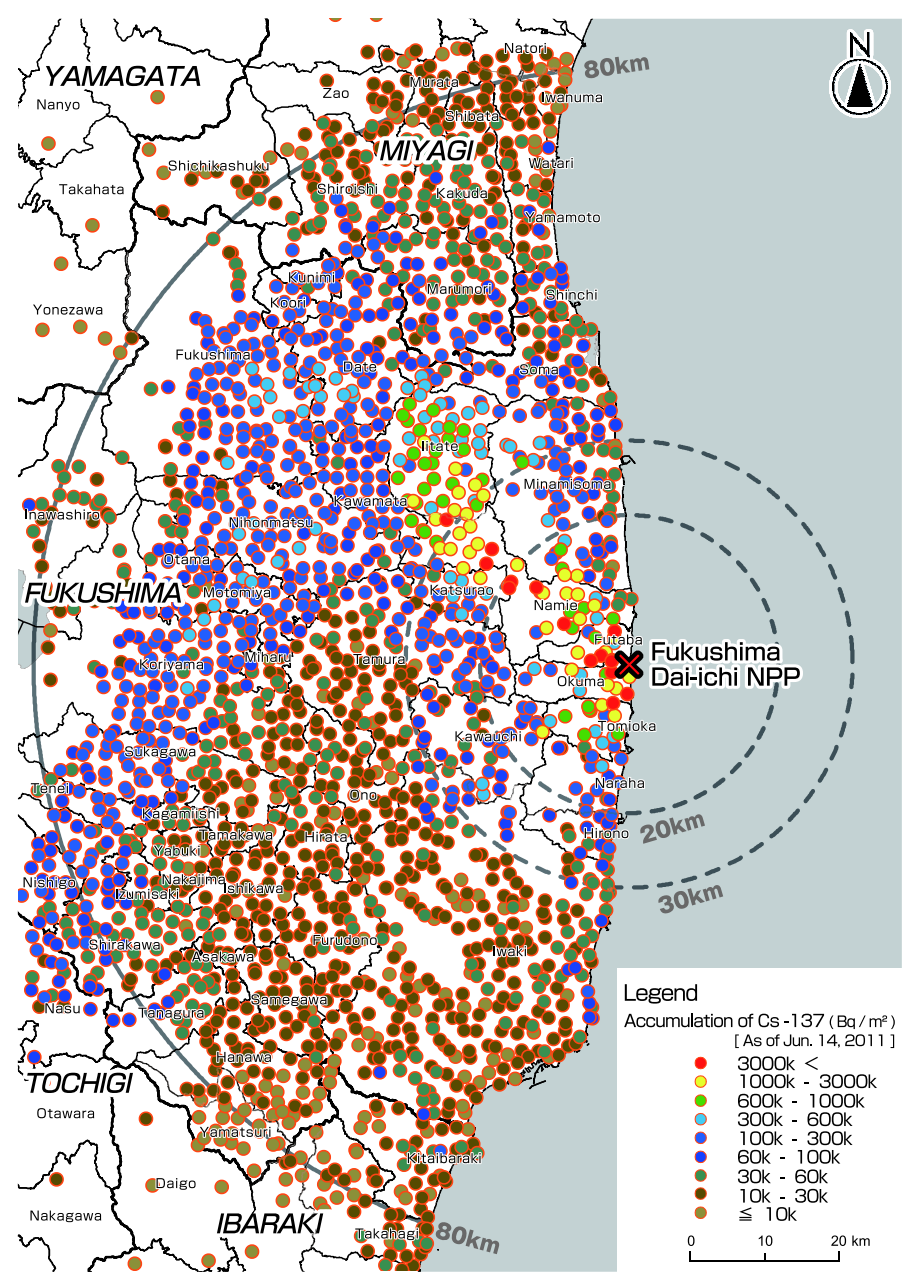

Figure 2. Contamination of soil due to ${ }^{137} \mathrm{Cs}$ per area. The unit is $\mathrm{Bq} / \mathrm{m}^{2}$.

ity measurement. The samples to be handled by university groups of nuclear physics and geoscience (nuclear chemistry) were sent first to the Center for Nuclear Study (CNS), the University of Tokyo, immediately after they were taken. The labels were checked, and missing informations (on the locations, etc.) were recovered as much as possible, which was not a trivial task at all. The samples were then sent to institutions over Japan. The standard of the actual measurement was controlled by the CNS also. The cross check was done between CNS and other institutions including JCAC. The final data have been stored in the data base which is accessible from public now, and have been visualized in the form of the contamination maps as shown in Figs. 1, 2 and 4 [1].

I shall overview the results of the survey. Figure 1 shows the radioactivity from ${ }^{134} \mathrm{Cs}$ per area. The actual unit is $\mathrm{Bq} / \mathrm{m}^{2}$. The color code is shown in the legend of the figure. One finds areas with higher contaminations in the north-west direction from the Fukushima Power plant. This is considered to be a combined effect of the wind and rain, as stated above. The radioactivity per area was found to be quite relevant, because materials containing Cs stick chemically to soil very strongly and stay almost 

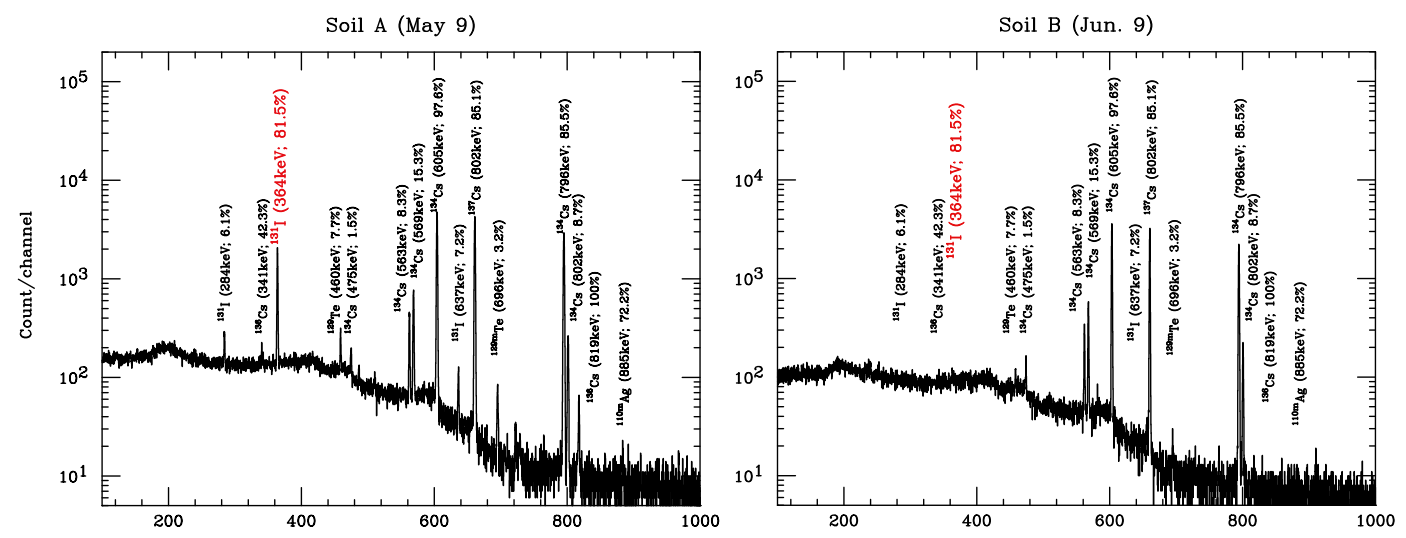

Figure 3. $\gamma$-ray spectra of soil samples taken in Fukushima prefecture. The measurement was carried out (left panel) on the 9th of May and (right panel) on the 9th of June. Note that $364 \mathrm{keV} \gamma$-ray from ${ }^{131} \mathrm{Xe}$ produced by the $\beta$ decay of ${ }^{131} \mathrm{I}$, shown in red, is seen on the left panel but not on the right. The isotopes shown are those before the $\beta$ decay.

within about $5 \mathrm{~cm}$ deep from the surface without being resolved into water. This means that the water contamination has stayed at very low level in general, which had made favorable consequences for agriculture.

Figure 2 shows the radioactivity from ${ }^{137} \mathrm{Cs}$ per area, in the same way as Fig. 1. One finds the same pattern of the contamination as the one by ${ }^{134} \mathrm{Cs}$. One of the most important feature in the systematic trends of contaminations by ${ }^{134,137} \mathrm{Cs}$ is the ratio between ${ }^{134} \mathrm{Cs}$ and ${ }^{137} \mathrm{Cs}$. The number of the decays per unit area per unit time (i.e., $\mathrm{Bq} / \mathrm{m}^{2}$ ) of ${ }^{134} \mathrm{Cs}$ is compared to that of ${ }^{137} \mathrm{Cs}$. This ratio turned out to be remarkably close to 0.92 over the whole region, if it is normalized to the 14th of June, 2011. Note that the half life is $2.07(30.08)$ years for ${ }^{134} \mathrm{Cs}\left({ }^{137} \mathrm{Cs}\right)$. This ratio has played a very important role to distinguish radioactive $\mathrm{Cs}$ isotopes produced by the Fukushima accident from those produced by atomic bomb tests by the USA, the USSR, the UK, France and China in the atmosphere. Since these tests have been carried out many years ago, ${ }^{134} \mathrm{Cs}$ is gone and only ${ }^{137} \mathrm{Cs}$ remains, although the radioactivity due to the bomb tests was very high in the past.

Another important outcome was the calibration of the data taken by the surface radioactivity measured in the air by car and by aircraft. Such measurements are much easier than the measurement of soil samples, but needs calibration. The calibration has been carried out for the soil sample and the measurement in the air at the sample location, which has increased the reliability of the measurements in the air.

The original intension of the survey included the measurement of the radioactivity from ${ }^{131} \mathrm{I} .{ }^{131} \mathrm{I}$ is potentially dangerous for the thyroid cancer of young generation. In Chernobyl, about 15 kids are reported to have died due to ${ }^{131}$ I contained in the milk from cows locally fed. Although milk production was forbidden in the Fukushima case immediately after the accident, the contamination of ${ }^{131}$ I was the highest priority to be measured. On the other side, there was rather long time interval between the proposal of the large-scale survey and the actual operation. The survey was carried out as government's official activity. Because even a handful of soil is a property of individual, local community or nation, the soil sample cannot be taken freely and the soil survey has to be endorsed officially. The bureaucratic process to approve the survey was quick by the government standard, but was not fast enough compared to the half life of ${ }^{131}$ I. Figure 3 shows $\gamma$ ray spectra taken on the 9 th of 


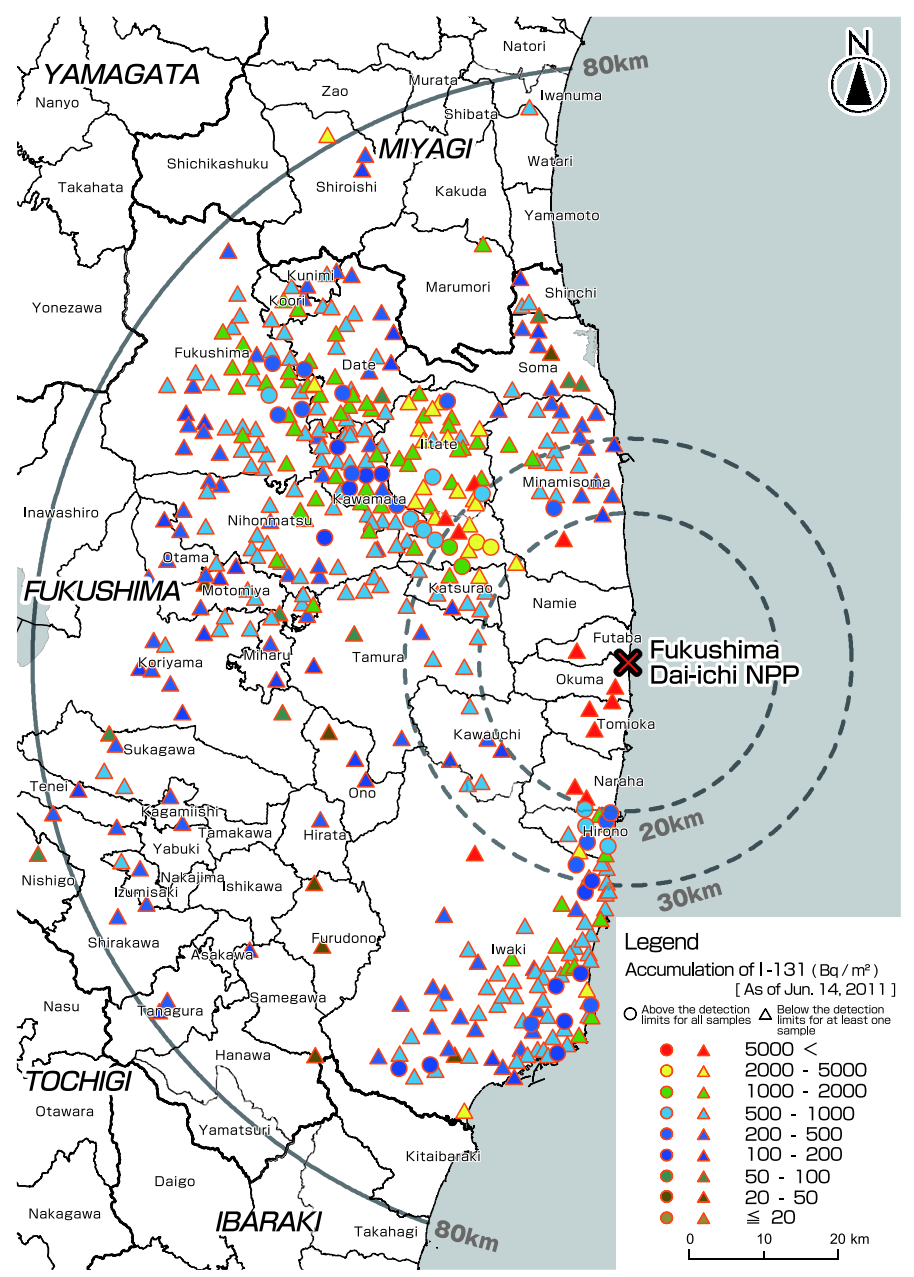

Figure 4. Contamination of soil due to ${ }^{131} \mathrm{I}$ per area. The unit is $\mathrm{Bq} / \mathrm{m}^{2}$.

May and on the 9th of June. One sees that the line at $364 \mathrm{keV}$ was strong on the 9th of May, but not visible a month later. The problem of layers of bureaucratic process for emergency operations may be a task to be solved or improved as soon as possible, certainly before the next severe accident of any kind.

Despite this difficulty, the measurement of ${ }^{131}$ I radioactivity was carried out, and the data have been taken in some locations. Figure 4 shows the radioactivity from ${ }^{131} \mathrm{I}$ per area, in the same way as Fig. 1. One finds higher contamination in the north-west direction and in the south direction along the coast. The latter direction differs from the south south-west direction of Cs isotopes, suggesting different leak timings and/or mechanisms between Cs and I isotopes.

As time has passed, the amount of ${ }^{131} \mathrm{I}$ has become much less than that of ${ }^{134} \mathrm{Cs}$ or ${ }^{137} \mathrm{Cs}$. These Cs isotopes produce Compton $\gamma$ rays which cover much lower $\gamma$-ray spectrum from ${ }^{131}$ I. Thus, even if the measurement is carried out for long time, one still cannot see clearly the peak of the 364-keV $\gamma$ ray from ${ }^{131} \mathrm{I}$. This is the reason why the contamination by ${ }^{131} \mathrm{I}$ has been measured with sufficient accuracy 
only for fewer locations than the Cs isotopes. Nevertheless, we have obtained meaningful result as shown in Fig. 4. We note that the data taken by the pilot survey performed early May 2011 exhibit distinct peaks of ${ }^{131} \mathrm{I}$, and that the observed radioactivity shows patterns of the contamination consistent to those shown in Fig. 4 [2]. Fortunately, the contamination by ${ }^{131} \mathrm{I}$ appears not to be of apparent immediate danger, but its consequences on the health of people, particularly young generations, must be kept watched for a long period.

Some nuclear physicists, including the CNS, are continuing the measurements of soil samples in a smaller scale.

\section{Summary}

I have presented a brief report on selected activities of Japanese nuclear physicists in response to the Fukushima Nuclear Power Plant accident.

Nuclear physicists are not experts of monitoring radioactivities of human being or environments like soil. However, they have high skill to measure radioactivities, ample knowledge on radioactivities and their sources, and are accustomed to safe handling of radioactive materials. They do not fear unnecessarily radioactivities. Usually, physicists are fond of behaving independently of anyone, and do not like controlled movements. At this accident, however, Japanese nuclear physicists got together to encounter difficulties and have made various coherent contributions in unfavorable working and living conditions. On the contrary, they did not receive credits on their own academic specialities. Although many other people contributed also in their own ways, I would like to stress that such purely voluntary and highly coherent actions are quite remarkable and deserve certain remembrance.

I feel at this moment that we, nuclear physicists, should think as to what can be done next, utilizing our knowledge, experience and skill. There should be some.

The author acknowledges many people for their collaborations in performing various activities in response to Fukushima Nuclear Power Plant accidents. For the preparation of this article, the author is particularly grateful to Prof. S. Shimoura of CNS and Mr. K. Tanaka of Japan Map Center.

\section{References}

[1] Final report in Japanese can be found at, summary: http://radioactivity.nsr.go.jp/ja/contents/6000/5233/view.html

details: http://radioactivity.nsr.go.jp/ja/contents/6000/5235/view.html

Its English version, summary : http://radioactivity.nsr.go.jp/en/contents/1000/294/24/PressR04\%200802s.pdf No detailed report in English is available.

[2] T. Otsuka, S. Shimoura, Y. Onda, A. Shinohara and T. Shibata, "Soil Radioactivity of ${ }^{131}$ I Measured by "Pilot Investigation" ", Radioisotopes (published by The Japan Radioisotope Association), 62, $752(2013)$. 
